\title{
EL LINEAMIENTO SANTA LUCÍA-AIGUÁ-MERÍN (URUGUAY): UN CORREDOR TECTÓNICO EXTENSIVO Y TRANSCURRENTE DEXTRAL PRECURSOR DE LA APERTURA ATLÁNTICA
}

\author{
EDUARDO A. ROSSELLO ${ }^{1}$, HÉCTOR DE SANTA ANA ${ }^{2,3}$ \& GERARDO VEROSLAVSKY ${ }^{3}$
}

\begin{abstract}
THE SANTA LUCIA-AIGUA-MERIN LINEAMENT (URUGUAY): AN EXTENSIONAL AND DEXTRAL WRENCHING CORRIDOR RELATED TO THE EARLY ATLANTIC BREAK-UPThe Santa Lucia-Aigua-Merin Lineament (SaLAM) is a $450 \mathrm{~km}$ long structural corridor running across the southeastern cratonic area of Uruguay, flanked by the shores of the Río de la Plata and Laguna Merín where two pull-apart depocenters (Santa Lucía and Laguna Merín basins) are developed. The SaLAM exhibits three segments, bounded by the Sarandi del Yí and Sierra Ballena N-S trending regional faults. The southwestern segment is represented by the Santa Lucía basin filled with $2,500 \mathrm{~m}$ Mesozoic volcanoclastic sediments. The intermediate segment is represented by an uplifted crystalline basement block that shows thin volcanosedimentary Mesozoic remains. The northeastern segment shows important alkaline magmatism and contains the Laguna Merin basin with Early Mesozoic volcanic rocks. The SaLAM was affected by two main tectonic phases: i) a Jurassic to Early Cretaceous extentional phase, represented by intraplate magmatism with few associated sediments, and ii) an Aptian-Albian dextral transtensive phase, with right-lateral strikeslip movement. The dextral strike-slip pattern is supported by the attitude of both volcanic and clastic elongated depocenters and contemporaneous with the Santa Lucía, Minas, Aiguá, Lascano and Velásquez troughs. Additional structural support comes from: i) the geometry of the brecciated fluorine and quartz veins located on the southern border of the Aiguá trough; ii) the rombohedrical shape of the main cretaceous depocenters; and iii) the geometry of the high gravimetric anomaly in the Laguna Merín basin. The genesis of the SaLAM is associated with an aborted rift, precursor of the Atlantic break-up. We suggest that the SaLAM was initially controlled by old structures of the Gondwana crystalline basement during the continental rifting followed by a second strike-slip tectonic phase associated with the drifting movement that led to the opening of the Atlantic. The integrated tectonic study of this corridor improves the stratigraphic correlations between the contemporaneous depocenters located at both extremes and support new economic perspectives related with the control on the ore and energy resources of the region.
\end{abstract}

Keywords: strike-slip tectonic, aborted rifting, Atlantic break-up, Mesozoic, Uruguay.

\begin{abstract}
RESUMEN El Lineamiento Santa Lucía-Aiguá-Merín (SaLAM) constituye un corredor estructural de dirección general NE con más de 450 $\mathrm{km}$ de largo que se extiende atravesando el área cratónica del sudeste del Uruguay desde la desembocadura del Río de la Plata hasta la Laguna Merín, donde desarrolla depocentros de tipo pull-apart (Laguna Merín y Santa Lucía). El SaLAM exhibe tres segmentos limitados por fallas regionales de dirección N-S (Sarandí del Yí y Sierra Ballena). En el segmento sudoccidental se emplaza la Cuenca de Santa Lucía, con más de $2.500 \mathrm{~m}$ de relleno sedimentario y volcánico mesozoico. El segmento intermedio está caracterizado por el levantamiento del basanento cris$2.500 \mathrm{~m}$ de relleno sedimentario y volcánico mesozoico. El segmento intermedio está caracterizado por el levantamiento del basamento de alcalino eocretácico y el emplazamiento de la Cuenca de Laguna Merín, rellena por materiales esencialmente volcánicos mesozoicos. El SaLAM muestra dos fases tectónicas principales en su evolución: i) una extensional, de edad Jurásico a Eocretácico representada por un magmatismo de intraplaca y escasa sedimentación asociada y; ii) una transtensional dextral, a partir del Aptiano-Albiano. El carácter transcurrente dextral se apoya en la disposición elongada de los depocentros volcánicos y sedimentarios sintectónicos a las fosas de Santa Lucía, Minas, Aiguá, Lascano y Velázquez. Se consideran evidencias adicionales, entre otras, i) la geometría de las brechas fluoríticos y venas de cuarzo localizadas en el borde sur de la fosa de Aiguá; ii) la geometría rombohedral de los principales depocentros cretácicos y; iii) la geometría de la fuerte anomalía gravimétrica que presenta la Cuenca Laguna Merín. El SaLAM es interpretado como un rift abortado precursor de la apertura Atlántica. Se propone que el desarrollo temprano del SaLAM, durante la ruptura, fue controlado por viejas direcciones estructurales del basamento cristalino gondwánico y una segunda fase tectónica, de tipo transcurrente, se vincula al inicio de la apertura Atlántica. La integración de los estudios tectónicos a lo largo de este corredor estructural permite establecer nuevas correlaciones estratigráficas entre los depocentros situados en ambos extremos y abre perspectivas económicas alternativas en el control de yacimientos y recursos energéticos de la región.
\end{abstract}

Palabras clave: tectónica transcurrente, rift abortado, apertura Atlántica, Mesozoico, Uruguay

\begin{abstract}
INTRODUCCIÓN
El presente trabajo describe la geometría y cinemática del Lineamiento Santa Lucía-Aiguá-Merín (SaLAM) vinculadas con el rifting juro-cretácico que afectó al basamento cristalino sudoriental de Uruguay. Este lineamiento estructural controló la sedimentación y el magmatismo Jurásico a Cretácico de la Cuenca Santa Lucía (CSL) y la Cuenca Laguna Merín (CLM), como la de los remanentes intermedios contemporáneos localizados en las fosas de Minas, Aiguá, Lascano y Velázquez. En su extremo sudoccidental, el SaLAM está temporoespacialmente vinculado a la génesis y desarrollo de la CSL y en el extremo nordoriental con la CLM con rellenos esencialmente volcánicos. En la porción central, se expresa por la disposición de remanentes y acumulaciones mesozoicas controlados por fallas normales dispuestas con rumbos comprendidos entre $\mathrm{N} 40^{\circ}$ a $45^{\circ}$ y $\mathrm{N} 100^{\circ}$ a $130^{\circ}$ comprendidos dentro de un corredor orientado ENE.
\end{abstract}

El SaLAM, cuya descripción preliminar fuera recientemente adelantada (Rossello et al. 1999b) se dispone con un rumbo noreste a lo largo de más de $450 \mathrm{~km}$ entre la costa del Río de la Plata, por el sudoeste, y la Laguna Merín, por el noreste (Fig. 1). De este modo, se sobreimpone a la estructuración heterogénea del basamento cristalino donde se individualizan tres grandes terrenos o unidades geotectónicas de edad precámbrica dispuestos submeridianalmente (Fig. 2): i) Piedra Alta, al Oeste, ii) Nico Pérez, al Centro y iii) Dom Feliciano o Cuchilla Dionisio, al Este (Bossi \& Campal 1992).
EL LINEAMIENTO SANTA LUCIA-AIGUA-MERIN

SaLAM constituye un corredor tectónico rectilíneo, de rumbo general $\mathrm{N} 70^{\circ}$, con funcionamiento extensional desde el Jurásico y transcurrente dextral durante el Cretácico tardío, que está expresado en sus extremos por las cuencas Santa Lucía y Laguna Merín y los afloramientos de rocas volcánicas, intrusivos y sedimentarios mesozoicos intermedios que se desarrollan próximos o en las fosas de Minas, Aiguá, Lascano y Velázquez (Bossi et al. 1998).

Para facilitar su descripción, la misma se realizará en tres segmentos (Fig. 1): sudoccidental (al oeste de la megafalla Sarandí del YíPiriápolis), intermedio (entre las megafallas Sarandí del Yí y Sierra Ballena) y nordoriental (al este de la megafalla Sierra Ballena).

Segmento sudoccidental Se describen aquí los registros mesozoicos que, asociados temporoespacialmente al SaLAM, se desarrollan al Oeste de la falla Sarandí del Yí-Piriápolis (Fig. 2), correspondientes al rift intracratónico de la CSL orientado con su eje mayor subparalelo a la disposición regional del mismo (Rossello et al. 1999a, Rossello et al. 2000). Esta se propagó a través de los principales planos de debilidad cortical del Terreno Piedra Alta, correspondientes con direcciones NNE preexistentes (Sprechmann et al. 1981, Santa Ana et al. 1994). La CSL, difiere desde el punto de vista espacial, de todas las demás zonas extensivas contemporáneas de esta porción del continente Sudamericano que resultaron en las

1 CONICET - Departamento de Ciencias Geológicas, UNIVERSIDAD DE BUENOS AIRES, Pabellón II, Ciudad Universitaria, 1428 - Buenos aires, Argentina. E-mail: rossello@ gl.fcen.uba.ar

2 ANCAP División Investigación y Desarrollo. Av. Lib. Gral. Lavalleja y Paysandú. CP 1090. MONTEVIDEO, Uruguay.

3 Departamento Geología (INGEPA), Facultad de Ciencias, UNIVERSIDAD DE LA REPUBLICA. Iguá 4225. CP 11400. Montevideo, Uruguay. E-mail: hdas@fcien.edu.uy, gerardo@fcien.edu.uy 


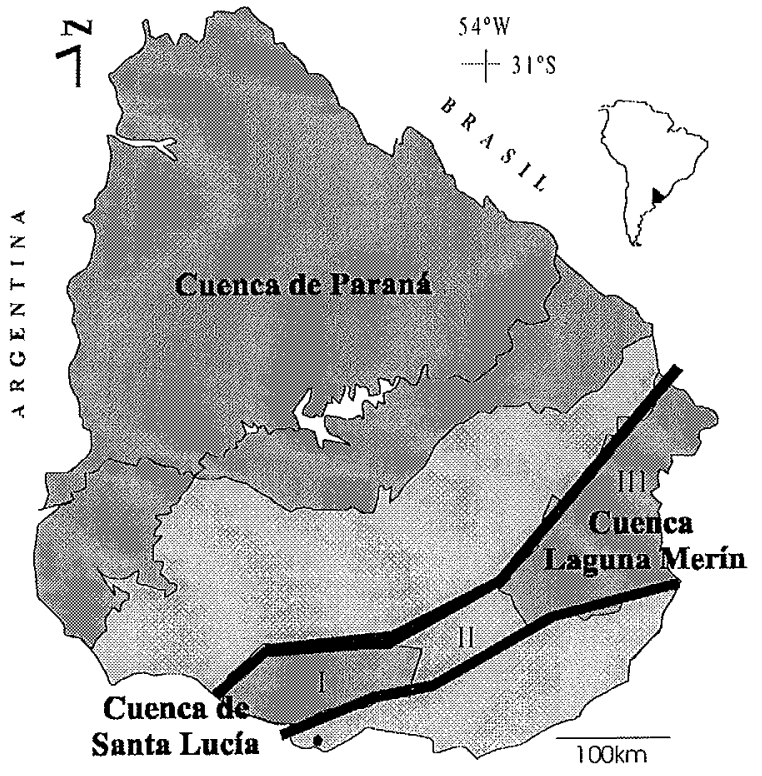

$35^{\circ} \mathrm{S}+\mathrm{F} 3^{\circ} \mathrm{W}$ NIODERAMLATA

Basamento cristalino Cuencas sedimentarias

Figura l - Localización relativa del Lineamiento Santa Lucía-Aigua-Merín sobre la porción sudoriental de la República Oriental del Uruguay. $I=S e g$. mento sudoccidental (Cuenca Santa Lucía); II = Segmento intermedio; III = Segmento nororiental (Cuenca Laguna Merín).

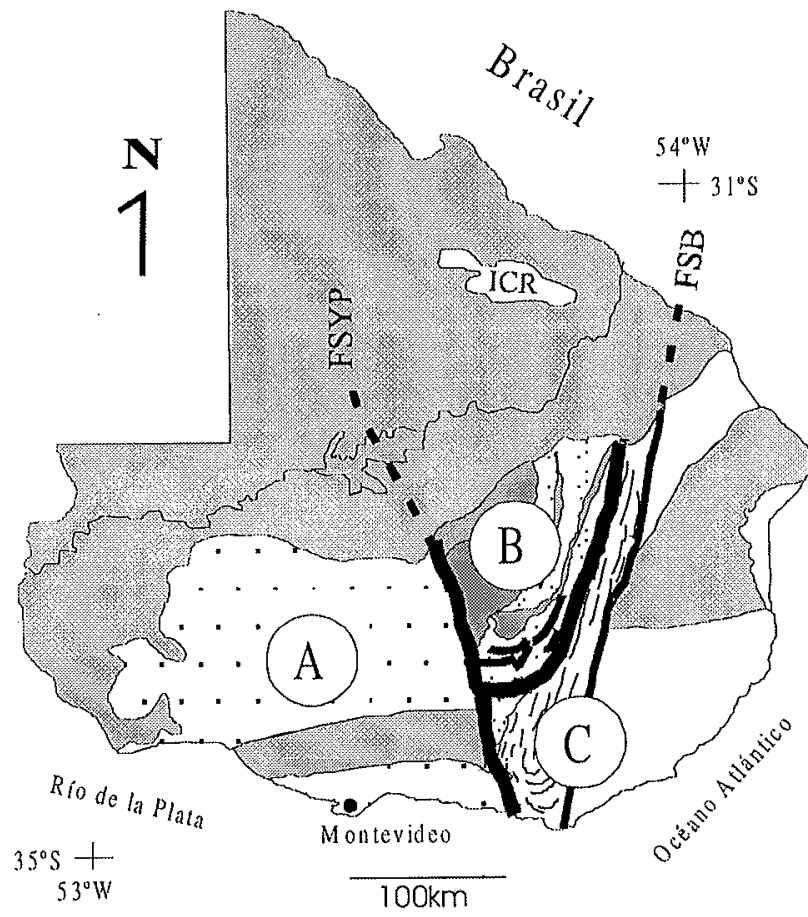

Figura 2 - Principales terrenos y rasgos tectónicos del basamento precámbrico de Uruguay. $A=$ Terreno Piedra Alta: $B=$ Terreno Nico Pérez; $C=$ Terrento Dom Feliciano o Cuchilla Dionisio; FSYP = Falla Sarandí Yí $F S B=$ Falla Sierra Ballena; ICR = "Isla Cristalina de Rivera" (modificada de Bossi \& Campal 1992).

cuencas Salado, Colorado, Punta del Este y Pelotas, porque exhiben una disposición transversal al margen continental (aulacogénica), tal como continuaron su evolución durante todo el Terciario recibiendo importante sedimentación.

La CSL muestra a lo largo de todo su desarrollo una sección asimétrica, conformada por un sistema conjugado de fallas normales lístricas de crecimiento sintéticas y antitéticas que definen grábenes y pilares tectónicos sucesivos de diferentes escalas (Rossello et al 1999a). Puede ser subdividida en dos fosas (Subcuenca Norte y Subcuenca Sur) que funcionaron, entre el Albiano y el Senoniano (Veroslavsky 1999), como ámbitos de sedimentación independientes separados por el Alto Santa Rosa (Fig. 3). Tiene una mayor definición en su margen austral expresada por el mayor funcionamiento de las fallas normales que lo limitan y el desarrollo restringido de las extrusiones volcánicas. Esta situación permite suponer que los fenómenos extensionales de apertura de la cuenca se ubican hacia el Este y que la extrusión de las volcanitas se produjo desde fisuras y diques de alimentación ubicados en los sectores más deprimidos de la cuenca.

Estudios realizados sobre datos geofísicos (gravimétricos y sísmicos), así como trabajos de relevamientos geológicos de superficie, caracterizan la asimetría de la cuenca, el patrón estructural y las diferencias petrotectónicas del basamento cristalino entre el borde Norte y el Sur (Veroslavsky 1999). Estos trabajos permiten soportar la hipótesis de la existencia de una discontinuidad principal a partir de la cual se nucleó y evolucionó el proceso de ruptura de la cuenca (Santa Ana \& Ucha 1994). Los relevamientos de superficie y subsuelo, el relleno volcano sedimentario típicos de la fases synrift (Leeder \& Gawthorpe 1987, Frostick \& Steel 1993) exhibe una arquitectura rombohedral que puede considerarse limitada por: i) Fallamientos dispuestos ENE-OSO que funcionaron como transcurrencias dextrales subverticales y que parecen haber sido controladas por las asimetrías primarias del basamento cristalino (en el sentido de Naylor $e t$ al. 1986); y ii) Fallamientos dispuestos sublatitudinalmente en posiciones acordes con fallamientos secundarios sintéticos de tipo Riedel y que corresponderían a estructuras extensivas lístricas de crecimiento que controlaron la geometría y disposición de los depocentros sedimentarios coetáneos.

De este modo, Rossello et al. (2000) caracterizan un depocentro típico de pull-apart como los definido en los trabajos de Mann et al. (1983) y Nielsen \& Sylvester (1995), que se exhibe compartimentado en dos depresiones con sus mayores profundidades adyacentes a las mayores estructuras y separadas por un alto intracuencal (Fig. 3), to-

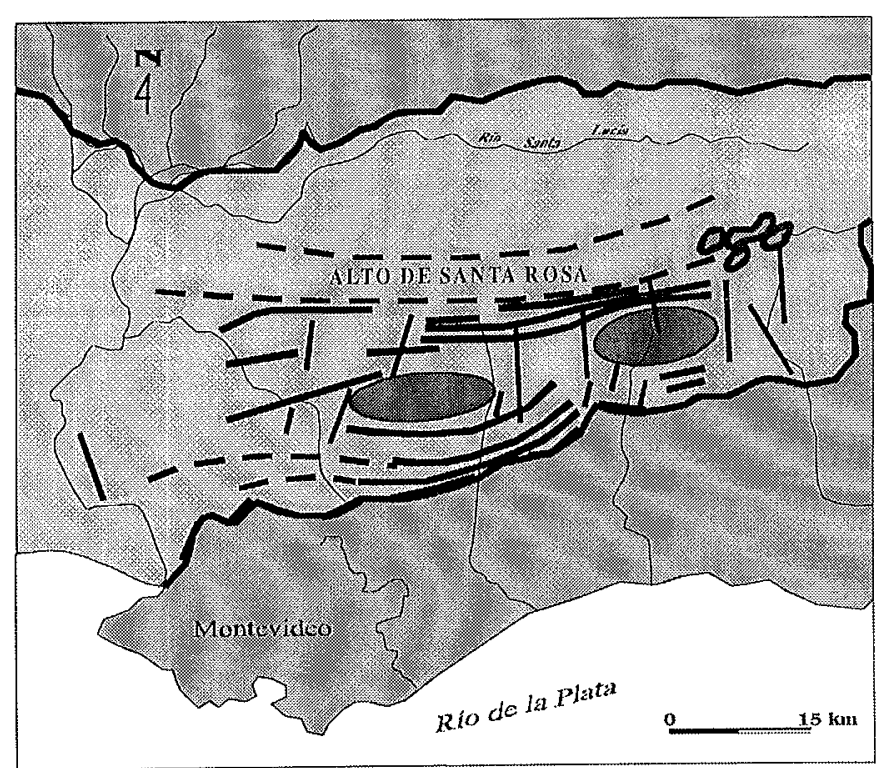

B asamento

Depocentros

\section{Clienca}

Fallas

Figura 3 - Esquema general de la compartimentación estructural de la Cuenca Santa Lucía y, en mayor detalle, de la Subcuenca Sur en el que se exhiben los dos depocentros sublatitudinales principales, a partir de relevamientos sísmicos y controles de pozos exploratorios (tomado de Veroslavsky 1999). 
dos dispuestos en échelon y con sus bordes más activos de tipo transcurrentes dextrales y otros pasivos de tipo normales de crecimiento.

Segmento intermedio En el segmento intermedio del SaLAM se comienza a delinear un leve alabeo en su traza de fractura principal que pasa progresivamente a direcciones más submeridionales hacia el oriente, adquiriendo un diseño sigmoidal en planta. Asociado al corredor se observan litologías y estructuras que se desarrollan integralmente en el Cinturón Dom Feliciano, entre las fallas de Sarandí del YíPiriápolis y Sierra Ballena (Fig. 4). El basamento exhibe anisotropías planares propias de su evolución metamórfica precámbrica (foliación, intrusiones graníticas, venulaciones, etc.) que se disponen preferencialmente con rumbos NE y NS y son, en consecuencia, cortadas por las estructuras distensivas vinculadas con el SaLAM.

Actualmente, la superficie del basamento aflora a unos $200 \mathrm{~m}$ s.n.m., razón por la cual sólo se reconocen remanentes mesozoicos aislados y delgados, preponderantemente volcánicos e hipabisales, en depresiones elongadas y estrechas limitadas por fallas normales sublatitudinales y protegidas en parte de la erosión que peneplaniza al cratón. Así, se reconocen unidades volcánicas extrusivas básicas y ácidas, representadas por los basaltos y riolitas con edades localizadas en torno a los 120 y $130 \mathrm{Ma}$ (Bossi \& Navarro 1991).

Los basaltos poseen textura microgranuda, a veces se presentan afaníticos, en general con vesículas rellenas por calcita, calcedonia, zeolitas, cuarzo y anhidrita. Ocurren en coladas no muy potentes que se disponen asociadas a pequeñas fosas: de dirección preferencial NE, como las situadas al norte de Minas y al noroeste de Villa Serrana (Fig. 4). Otras fosas pequeñas y elongadas que muestran un notable control estructural de dirección $\mathrm{N} 100^{\circ}$ y $130^{\circ}$ son las situadas al noroeste de Mariscala y al sudeste de Colón. En todas, es común que las coladas de basaltos se dispongan directamente sobre áreas de basamento.

Las riolitas son de tipo porfírica y están asociadas a brechas riolíticas e ignimbritas que se apoyan y recortan a los basaltos, constituyendo una sucessión volcanioclástica no muy potente aunque con desarrollos preferenciales en dirección NE (Fig. 4). En la región de Arequita, donde ésta sucesión sé apoya sobre los basaltos, está afectada por fallas de rúmbo $\mathrm{N} 70^{\circ}$ con componente dextral conjugadas con otras fallas normales dispuestas con rumbos $\mathrm{N} 140^{\circ}$ (Fig. 5). También, en la zona del Arroyo El Perdido al NE de Minas (Fig. 4), dentro de conglomerados con textura matriz sostén típicos de ambientes de synrift (Formación Cañada Solís, Rossello et al. 2000) se reconocen grietas lenticulares de cuarzo lechoso de un metro de largo por $1 \mathrm{~cm}$ de potencia dispuestas subverficalmente con rumbos $\mathrm{N} 110^{\circ}$ que exhiben arreglos en trenes escalonados en bandas con rumbo $\mathrm{N}$ $80^{\circ}$ determinantes de desplazamientos transtensivos dextrales.

Segmento nordoriental Al Este del megafracturamiento Sierra Ballena (Fig. 2), se desarrolla el segmento nordoriental del SaLAM, el que sufre una clara inflexión para disponerse con rumbos $N 50^{\circ}$ a $45^{\circ}$, a partir del Lineamiento Cebollatí-Merín y $\mathrm{N} 60^{\circ}$, Lineamiento AiguáIndia Muerta-Chuy que controlaron el desarrollo extensional de la CLM (Fig. 6). Próximo a la estructura Sierra Ballena, hacia el Este, se desarrolla otro conjunto de pequeñas fosas rellenas por basaltos, andesitas, riolitas y dacitas (Lascanó, Aiguá y Treinta y Tres) así como las rocas intrusivas, hipabisales y volcánicas alcalinas que conforman la Formación Valle Chico en el sentido de Pirelli (1999), de edad eocretácica (Preciozzi et al. 1985, Pirelli 1999, Muzio et al. 1999). La Formación Valle Chico reúne a sienitas, granitos, traquitas y riolitas alcalifeldespáticas, así como, subordinadamente, pórfidos y vidrios riolíticos y filones de cuarzo (Pirelli 1999). Este complejo ígneo, con expresión superficial subcircular de $250 \mathrm{~km}^{2}$ (Muzio \& Artur 1998), está afectado por una frácturación intensa cuya dirección preponderante es NO (Pirelli 1999) coincidiendo también con la dirección de diques y filones que recortan a la unidad.

Al Este de la fosa de Aiguá, próximo a la localidad de Paso de los Talas y sobre el contacto entre el basamento granítico y vulcanitas ácidas, hacia el Oeste, se desarrolló un conjunto de fracturas rellenas por fluorita (Bossi 1978) que fueron temporariamente explotadas con el nombre de Mina Florencia (Fig. 7). La mineralización es de edad Cretácica, se encuentra limitada y controlada al norte de la falla normal de Aiguá, de rumbo $\mathrm{N} 60^{\circ}$, que comenzando al sur de Aiguá se extiende por varias decenas de kilómetros hacia Paso de los Talas (Gómez-Rifas \& Pérez-Peirano 1992). La mineralización se presenta en filones decimétricos dispuestos saltuariamente a lo largo de $1,8 \mathrm{~km}$ con cuerpos vetiformes de hasta $0,5 \mathrm{~m}$ de potencia en clara asociación paragenética con filones de cuarzo, baritina, epidoto y manganita (Fig. 7). Esta mineralización constituye facies brechosas muy características y ocasionalmente, en estructuras bandeadas finas a gruesas. Se la interpreta, en forma preliminar, como precipitadas de soluciones hidrotermales enriquecidas por la lixiviación del flúor asociadas a la actividad magmática sieno-granítica tardía del complejo Valle Chico. Es importante señalar que, tanto los filones de cuarzo como los fluoríticos, muestran una disposición en planta en forma de sigmoidal, preferencialmente con dirección NE hasta E-O e inclinaciones comprendidas entre la vertical y los $80^{\circ}$ hacia el Norte. Bosse et al. (1982) indicaron originalmente que la deformación pudo haberse generado a partir de la actuación de movimientos parciales horizontales a lo largo de la falla de borde de la fosa en dirección $70^{\circ}$ E y reconocen una fase de deformación sin a postectónica al proceso hidrotermal que diera origen a los filones fluoríticos y cuarzosos.

Desde el punto de vista cinemático, la disposición espacial de estos filones dentro del corredor definido por el SaLAM remarcan situaciones dilatantes coherentes con un funcionamiento dextral regional (Ramsay \& Huber 1983). Además, pueden correlacionarse estas manifestaciones minerales con las semejantes existentes más al norte, en Brasil, donde se reconocen filones de fluorita encajados en antiguos lineamientos Brasilianos orientados NNE-SSO reactivados durante el Cretácico como fallas transcurrentes y normales (Dardenne et al. 1997). Los filones fluoríticos con contenidos de tierras raras que permiten asociarlos a cuatro fases de mineralización principales con tres primeras ocurridas entre los 140 y $100 \mathrm{Ma}$ y una cuarta en torno a los $70 \mathrm{Ma}$ vinculados a lixiviaciones del flúor por circulaciones hidrotermales relacionados a anomalías térmicas debidas a la apertura del Atlántico austral o directamente a rocas alcalinas (Dardenne 1999).
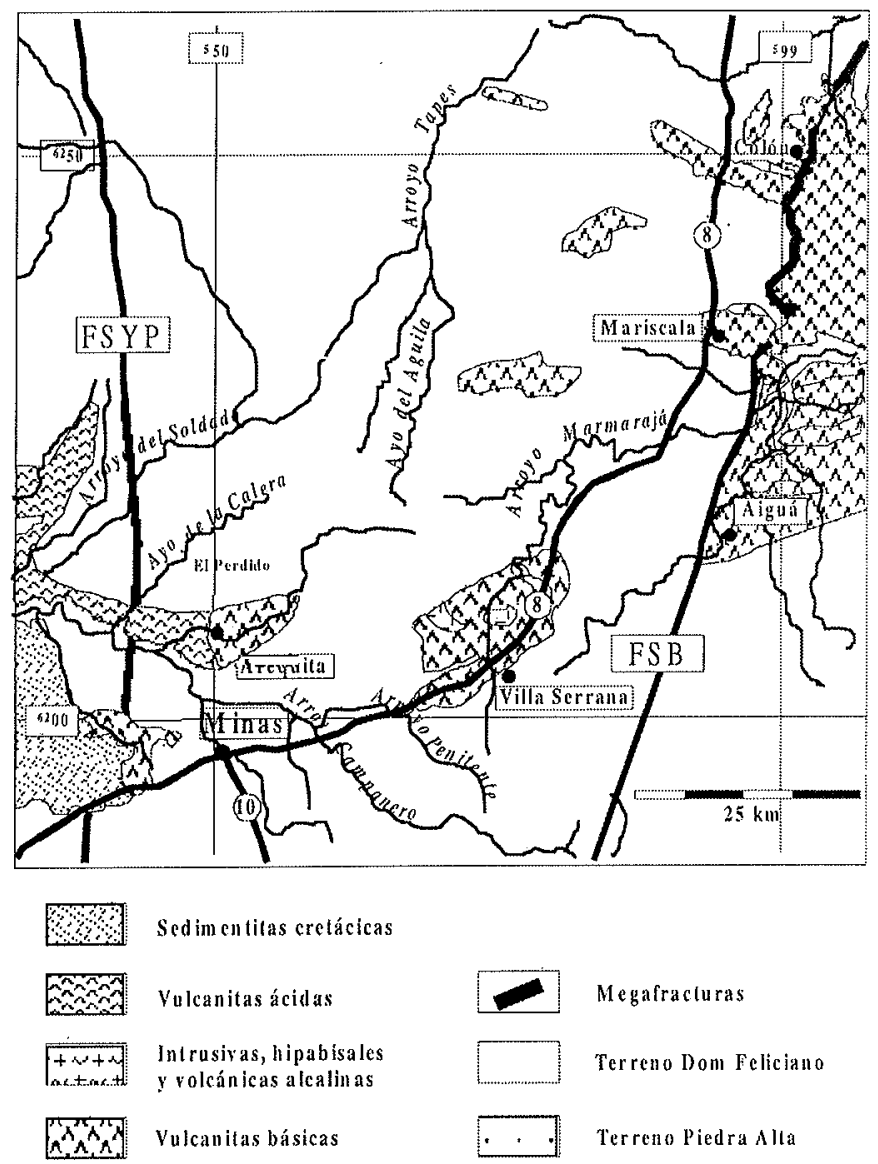

Figura 4 - Detalle de los afloramientos de rocas volcánicas cretácicas en la porción central del corredor SaLAM, limitado entre las megafracturas Sarandi del Yi (FSYP) y el de Sierra Ballena (FSB) (tomado de Veroslavsky 1999). 


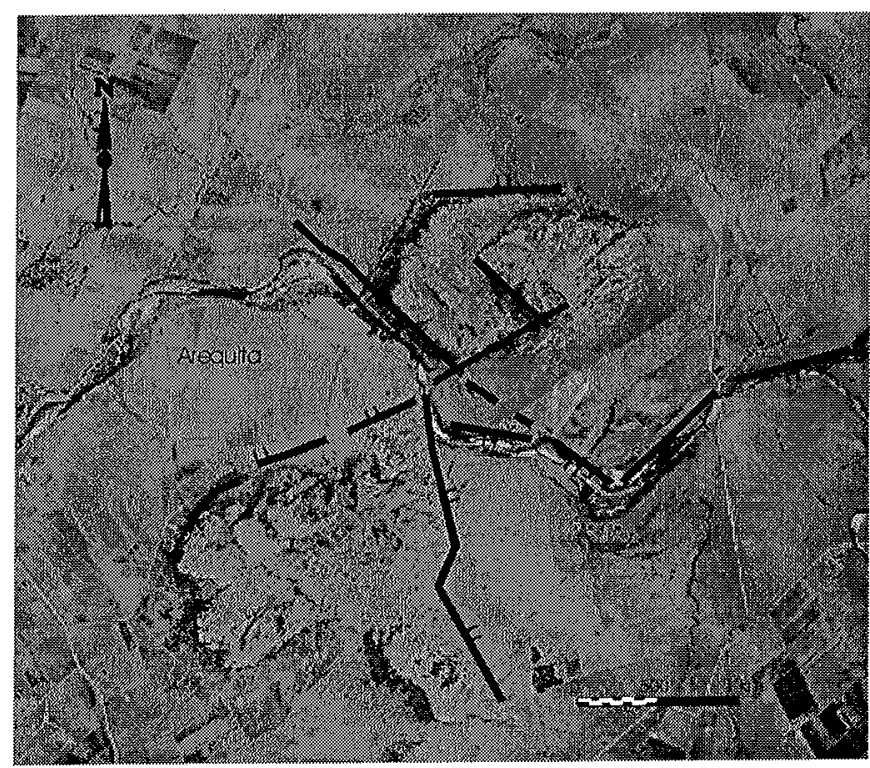

Figura 5 - Fotografía aérea de la comarca de Arequita donde se observan las volcanitas de la formación homónima y los fracturamientos principales que las afectan.
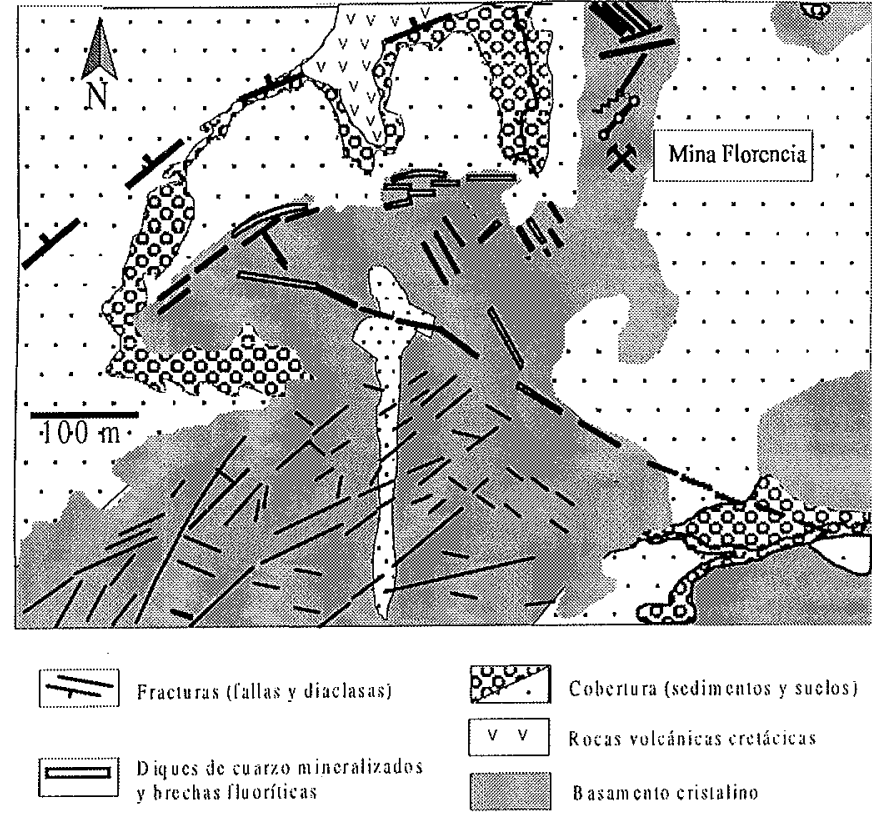

Figura 7 - Esquema geológico del sector de las vetas de fluorita de Mina Florencia emplazada sobre el contacto entre el basamento al sur y volcanitas cretácicas al norte (Simplificada de Bosse et al. 1982).

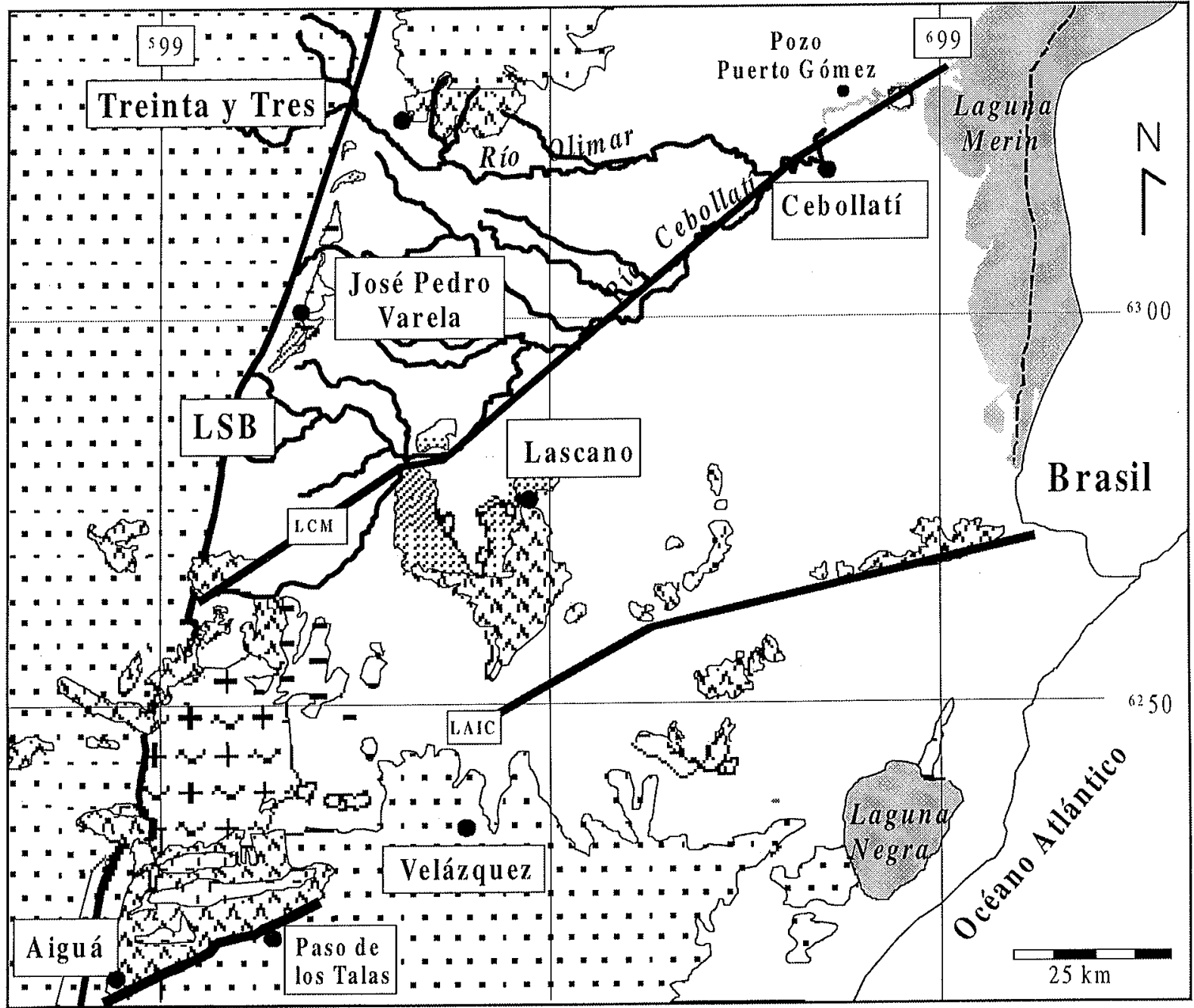

Figura 6 - Esquema geológico del sector nordoriental del SaLAM, al este del megafracturamiento Sierra Ballena (LSB), próximo al límite con Brasil. Las trazas corresponden a las indicadas en la Fig. 4. 
El ámbito de la CLM es un área casi totalmente cubierta por sedimentitas cenozoicas y con la expresión máxima del magmatismo básico cretácico (Fig. 6). Este está representado por más de $1.000 \mathrm{~m}$ de potencia de basaltos y andesitas microgranudas y con estructura amigdaloide (Formación Puerto Gómez, según Caorsi \& Goñi 1958) que recorta el pozo Puerto Gómez $N^{\circ} 502$ (entre -212 y - $1.170 \mathrm{~m}$ bbp) y que no llegó a basamento (Bossi et al. 1998). El análisis geoquímico de los registros de esta perforación permitió a Gómez-Rifas \& Masquelín-Arcelus (1996) interpretar que los últimos 400 m pueden ser asociados, por su composición química, a rocas correspondientes al fondo oceánico.

El sector ocupado por la CLM registra la anomalía gravimétrica positiva de Bouguer más importante del territorio continental del Uruguay que llega a valores superiores a los $60 \mathrm{mGal}$ (Fig. 8). De este modo, contrasta fuertemente con el comportamiento gravimétrico regional del resto del basamento substancialmente grueso de Uruguay (litosfera estimada en $110 \mathrm{~km}$ de espesor, según Introcaso \& Huerta 1982). En la plataforma continental uruguayo-brasileña (Cinturón Dom Feliciano), se han calculado espesores de corteza continental en torno a los $20 \mathrm{~km}$, pudiéndose observar a través de las líneas sísmicas de refracción un adelgazamiento en dirección al mar que permiten estimar espesores de corteza continental de sólo $10 \mathrm{~km}$, ya próximo al contacto con la corteza oceánica (Fontana 1990).

Se interpreta a esta anomalía como la respuesta de mayor densidad de una gran masa de material de origen mantélico que puede expresar la generación de una protocorteza oceánica por la presencia de los derrames basálticos. Por otro lado, esta anomalía permite reconocer en planta una forma ligeramente rombohedral, limitada por los lineamientos Cebollatí-Merín y Aiguá-India Muerta-Chuy (Fig. 6) que sugiere una morfología semejante a un pull apart (Mann et al. 1983), con una geometría y disposición alongada en la dirección $\mathrm{N} 70^{\circ}$, que indica transtensión dextral (Christie-Blick \& Biddle 1985), tal como se esquematiza en la Fig. 9.

INTERPRETACIÓN TECTÓNICA DEL SaLAM Se pueden reconocer dos fases tectónicas, en la evolución del SaLAM: i) una inicial Juro-eocretácica, claramente distensiva; y ii) otra posterior, a partir de Aptiano-Albiano, donde se establece el carácter transcurrente dextral. El abandono de la extensión más pura del SaLAM debe haberse generado, de modo transicional, por una mayor eficiencia mecánica de las antiguas discontinuidades submeridianales localizadas hacia el oriente del terreno Dom Feliciano que pudieron haber canalizado la apertura y creación de la corteza oceánica Atlántica (Fig. 10).

Fase inicial (distensión) La configuración paleogeográfica del Paleozoico de la región indica que el Cratón Río de la Plata fue, en esos tiempos, una región alta desprovista de cobertura sedimentaria (França et al. 1996). Sin embargo, una vez que comenzó a operar el rifting relacionado con la apertura Atlántica (Uliana \& Biddle 1988, Rossello \& Mozetic 1999) y a evolucionar las cuencas Juro-Cretácicas de la región del Plata comenzaron a individualizarse los altos intracuencales de: i) Martín García, que limita las cuencas del Salado y Punta del Este; ii) Polonio, que constituye el límite entre las cuencas Punta del Este y Pelotas; iii) del Plata (Dalla Salda et al. 1988), que limitó parcialmente las cuencas Punta del Este y Santa Lucía; y iv) Colonia-Mercedes, que separa la Cuenca Santa Lucía con la Cuenca Norte o Paraná (Santa Ana et al. 1994).

El SaLAM comienza a expresarse como una zona extensiva desde el Jurásico tardío directamente vinculada con la megafragmentación del Gondwana desarrollada sobre el Cratón La Plata (Sprechmann et al. 1981, Dalla Salda et al. 1988, França et al. 1996) a lo largo de antiguas zonas de debilidad del mismo. De esta manera, compartió una historia tectónica y volcánica común con las cuencas Santa Lucía y Laguna Merin, localizadas sobre la misma zona de megafractura.

El principal carácter extensional del SaLAM en la corteza gondwánica está indicado también por la presencia de magmatismo de intraplaca a lo largo de todo su recorrido. Así, su extremo oriental se puede asociar con los potentes niveles de basaltos y litologías volcaniclásticas jurásicas $y$, en su segmento central, con el magmatismo alcalino de Valle Chico y los basaltos y riolitas presentes en fosas del segmento intermedio. La geoquímica caracteriza a este magmatismo como alcalino transicional de ambiente de intraplaca (Muzio \& Artur 1998) y probablemente con mayor contaminación cortical, según los valores geoquímicos obtenidos por Gómez-Rifas \& Masquelin-Arcelus (1996). Por otro lado, este volcanismo guarda estrechas relaciones temporales, evolutivas y geoquímicas con las extensas extrusiones basálticas y riolíticas de la vecina Cuenca del Paraná (Cuenca del Norte, según Santa Ana 1989) descriptas recientemente por Peate (1997), llamativamente dispuesta también con un depocentro regional elongado de modo subparalelo al SaLAM y al borde Atlántico (Turner et al. 1994).

Los valores fuertemente anómalos de la gravimetría en la CLM estarían relacionados con posibles diques o fuentes de alimentación mantélica que determinaron cuerpos ultramáficos asociados con relictos de suelo oceánico que contrastan con el marcado carácter continental del basamento.

En el ámbito de la Cuenca Santa Lucía esta primera reactivación tuvo un carácter menos expresivo, ya que está representado por sucesivas y delgadas coladas de basaltos (148 $\mathrm{m}$ de potencia máxima en el pozo Piedra Sola) y sedimentación clástica aluvial (Formación Cañada Solís). Ambas anomalías, están claramente aisladas de las conocidas en ámbitos oceánicos y marginales del continente sudamericano (Rabinowitz \& LaBrecque 1979) por un alto estructural que sobre el continente lo representa el terreno Dom Feliciano.

Con respecto a la presencia del magmatismo es importante remarcar que en la CLM fue más activo e importante ya que al menos 1.000 $m$ de basaltos fueron registrados mientras que en la CSL supera tan sólo $100 \mathrm{~m}$ de espesor. Esta fuerte variación podría justificarse a partir de la necesidad de compensar isostáticamente los adelgazamientos corticales. Por otro lado, el recorrido del magma de origen mantélico dentro de espesores diferentes de corteza cortical pudo haber influido en las fuertes variaciones geoquímicas que ellos presentan.

Fase tardía (transcurrente) El funcionamiento del SaLAM como una transcurrencia dextral estaría relacionado a la actuación de esfuerzos de alivio de intraplaca vinculados al inicio de la fragmentación del Gondwana y a la fase de deriva de la placa Sudamericana a partir del Aptiano. Al respecto, varios modelos señalan los procesos de apertura atlántica que proponen un régimen de esfuerzos que supone la actuación de zonas con transcurrencias dextrales que se desarrollan sobre discontinuidades precámbricas (Uliana \& Biddle 1988, Conceição et al. 1988, Nurmberg \& Muller 1991, Urien et al. 1995, entre otros).

El carácter transcurrente dextral del SaLAM está determinado, a escala regional, por el arreglo espacial escalonado de diferentes rasgos extensivos dispuestos con rumbos $\mathrm{N} 100^{\circ}$ a $130^{\circ}$ a lo largo del corredor $\mathrm{N} 70^{\circ}$ : i) los depocentros y geometría de sedimentitas y de efusiones volcánicas contemporáneas con el funcionamiento JuroCretácico de la CSL (véanse mayores detalles en Veroslavsky 1999, Rossello et al. 1999b); ii) el arrumbamiento de los ejes longitudinales sublatitudinales a ESE-ONO de las fosas estrechas limitadas por fallas normales y con relleno volcanosedimentario de Minas, Aiguá, Colón, Lascano (Fig. 1); y iii) la deformación de los filones fluoríticos y cuarzosos (Bosse et al. 1982), en el margen austral de la fosa Aiguá; iv) intensa fracturación distensiva NO del complejo Valle Chico (Pirelli 1999).

Los diques de basaltos se disponen en cuerpos subverticales con rumbos $\mathrm{N} 100^{\circ}$ y normales a la dirección principal $\mathrm{N} 70^{\circ}$ del SaLAM, al igual que la localización especial de la fracturación y filones NO de Valle Chico. Estos arreglos espaciales indicarían fallamientos secundarios que a partir de las direcciones que exhiben, se los puede interpretar, siguiendo a Ramsay \& Huber (1983), como sintéticas de tipo Riedel $\left(\mathrm{N} 100^{\circ}\right.$ ) y antitéticas de tipo anti Riedel (NO), siguiéndose los arreglos espaciales y nomenclaturas de Christie-Blick \& Biddle (1985), Harding (1990), Thurston \& Theiss (1991) y Woodcock \& Schubert (1994).

Por otro lado, el SaLAM presenta pandeos o curvamientos locales a lo largo de toda su extensión. De este modo, se puede comprobar que en el segmento intermedio exhibe su plano principal ligeramente rotado hacia posiciones más submeridionales lo que generó un pandeo constrictivo que fue el responsable del abovedamiento del basamento cristalino. Contrariamente, hacia sus extremos, la dirección principal del corredor transcurrente tuerce hacia posiciones más latitudinales determinando pandeos de alivios (releasing bend) de modo tal que provocó las condiciones extensivas que facilitaron la generación del rifting de las cuencas Santa Lucía y Laguna Merín. En este sentido, la Fig. 9 sintetiza la cinemática transcurrente propuesta para explicar el funcionamiento del SaLAM durante el Mesozoico como una zona de precursora de la fragmentación del continente Gondwana que final- 


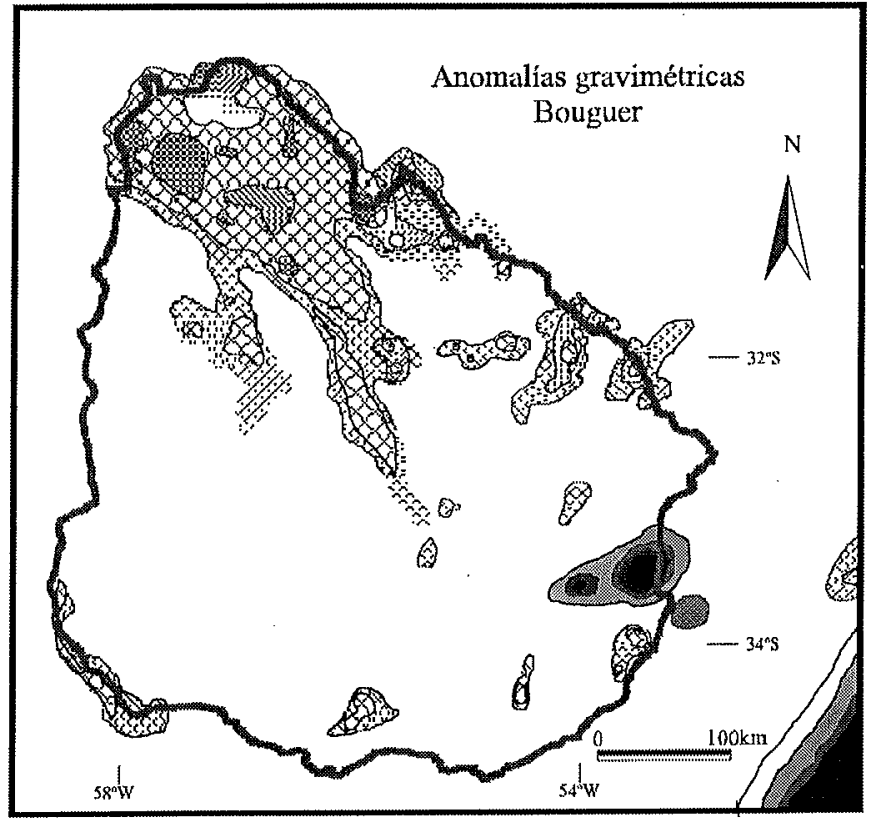

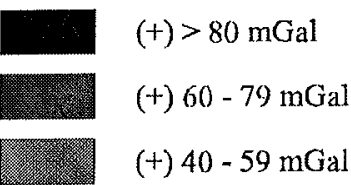

Figura 8 - Mapa de anomalias gravimétricas de Bouguer de la República Oriental del Uruguay. Nótese la importante anomalía de más de $60 \mathrm{mGal}$ localizada en la Cuenca Laguna Merín (Carta Gravimétrica Provisoria de la República Oriental del Uruguay, fuente: Servicio Geográfico Militar 1973).

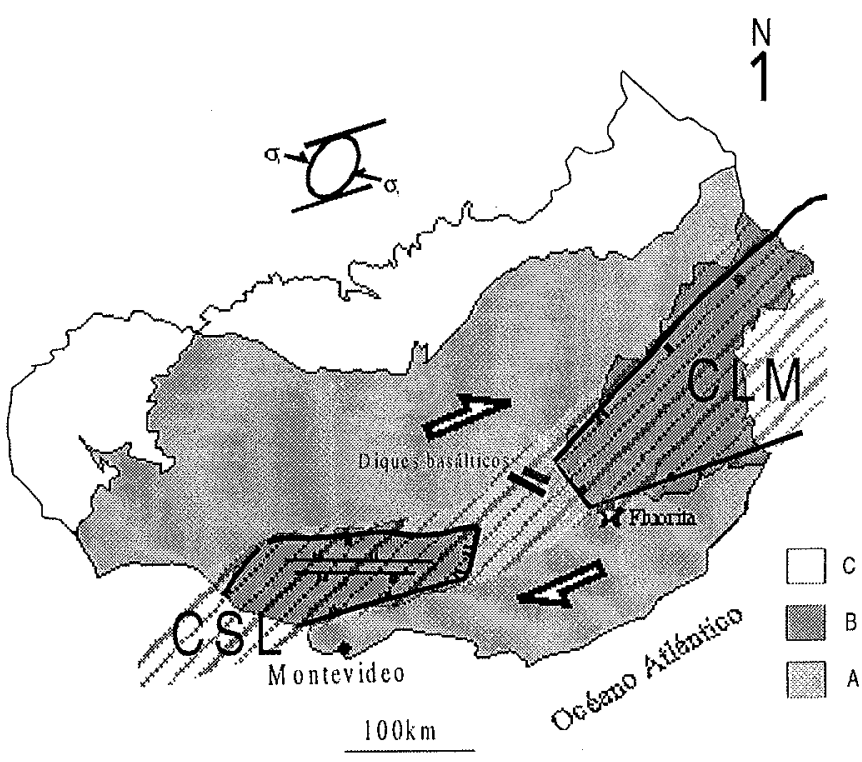

Figura 9 - Esquema cinemático transcurrente dextral del Lineamiento SaLAM expresado por el rayado diagonal donde se resaltan con trazos continuos la posición relativa de las principales discontinuidades. $A$, indica los afloramientos de basamento cristalino, $B$, indica la posición de los depocentros juro-cretácicos contemporáneos con el SaLAM: Cuenca Santa Lucia (CSL) en el extremo sudoccidental y de la Cuenca Laguna Merín (CLM) en el extremo nordoriental y $C$, indica los afloramientos de la Cuenca del Norte. El segmento intermedio se encuentra casi desprovisto de sedimentación juro-cretácica por su carácter transpresivo que controla el emplazamiento de diques basálticos (remarcados con trazos gruesos) que siguen fracturas extensionales dispuestas paralelamente a la dirección de compresión, tal como se expresa en el diagrama inserto. La estrella indica la posición de las vetas de fluorita (ver fig.7). mente se localizó sobre la posición actual del Océano Atlántico (Fig. $10)$.

Finalmente, durante el Cenozoico, a partir de información de subsuelo se pueden reconocer fenómenos de reactivación tectónica que invierten algunas estructuras distensivas (Rossello et al. 1999) siguiendo patrones acordes con la deformación global para toda Sudamérica (Cobbold et al. 1996). Estos esfuerzos andinos pueden ser los responsables, al menos parciales, que el segmento intermedio del SaLAM (Fig. 1) haya ascendido diferencialmente como un pilar tectónico con respecto a los vecinos, razón por la cual, este tramo, exhibe solamente retazos volcánicos basales apoyados sobre el basamento (Fig. 4).

CONCLUSIONES Se considera al SaLAM como la expresión geotectónica de una zona extensiva cortical con una llamativa disposición subparalela al desarrollo temprano de la apertura Atlántica y del depocentro longitudinal de la vecina Cuenca Paraná a través del continente Gondwana. Sin embargo, el funcionamiento extensional posterior del rifting que expresa este lineamiento se vio superado por otro localizado más al oriente y que efectivamente originó al actual Océano Atlántico.

Se reconoce un particionamiento estructural a lo largo del SaLAM,

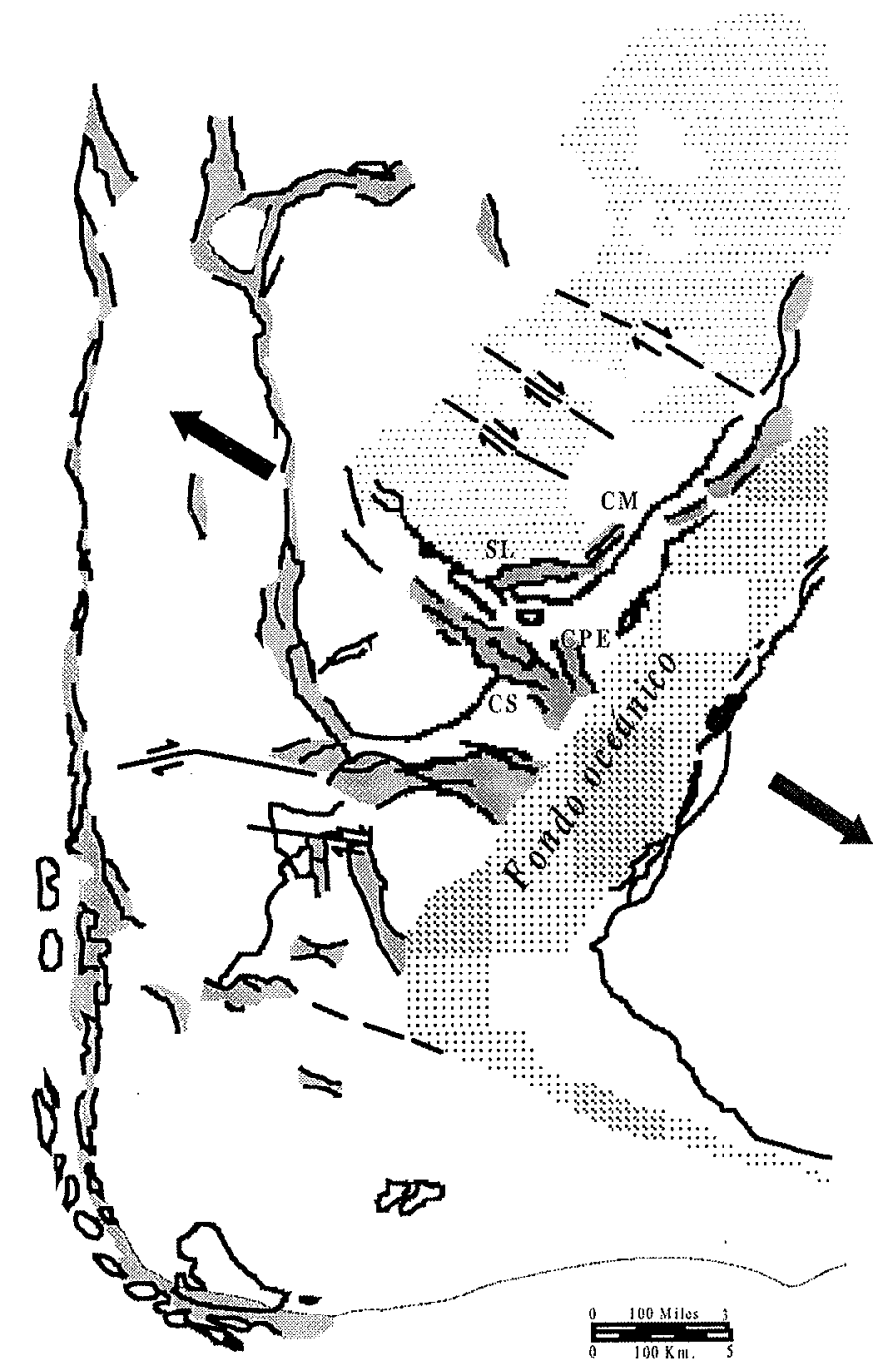

Figura 10 - Esquema tectónico del extremo austral del continente sudamericano durante el Cretácico (tomado de Uliana \& Biddle 1988). SL: Cuenca Santa Lucía. CS: Cuenca del Salado. CPE: Cuenca Punta del Este. CM: Cuenca Laguna Merín. Las flechas indican la dirección de divergencia relativa entre Sudamérica y Africa. Las zonas grisadas obscuras indican la posición de corteza oceánica, las zonas grisadas claras indican la posición de depocentros sedimentarios y las zonas punteadas indican la posición de las efusiones basálticas de la Formación Serra Geral. 
expresado por un segmento central transpresivo (sector intermedio) y otros eminentemente transtensivos hacia los dos extremos con depocentros tridimensionalmente asimétricos. El carácter transcurrente dextral controló el desarrollo de las fases de verdadero rift de los numerosos depocentros que resaltan la traza del SaLAM y que están limitados por fallas normales de crecimiento con rechazos verticales importantes, que en el caso de la CSL su mayor actividad es concomitante con la sedimentación albiana de tipo synrift (formaciones Castellanos, Migues y Cañada Solís, parcialmente).

El SaLAM está directamente vinculado con: i) la generación de depocentros subparalelos al margen Atlántico de tipo pull-apart (con relleno esencialmente sedimentario en la CSL y magmático en CLM) sobre un dominio cratónico preestructurado; ii) la existencia de derrames basálticos tempranos de muy escaso desarrollo asociados a las estructuras limitantes de la cuenca, sucedidas hacia su extremo oriental por erupciones riolíticas; iii) el desarrollo de un escenario tectosedimentario transtensivo dextral asociado a una fase synrift principal de edad Albiana (fundamentalmente en el Este y Sudeste) con sedimentación de lutitas negras fosilíferas con niveles evaporíticos correspondientes a ámbitos lacustres restringidos y la falta de registros marinos con traslapes senonianos; y iv) la existencia de estructuras de inversión de funcionamiento transpresivo relacionadas a episodios tectónicos andinos tardíos.

Desde el punto de vista económico, la presencia de magmatismo intenso vinculado temporoespacialmente con estructuras dilatantes sublatitudinales a NNO-SSE, ofrece interesantes objetivos prospectivos mineros por la posibilidad de generación, circulación y control de fluidos hidrotermales que podría incrementar al actual conocimiento de los recursos naturales de la región (Gómez-Rifas \& Pérez-Peirano 1992). Por otro lado, se pueden reconocer direcciones preferenciales de extensión dentro del ámbito cristalino en las que podrían prospectarse cuerpos intrusivos alcalinos intracratónicos uniextendidos semejantes a los importantes y explorados intensamente en comarcas comparables de Brasil (Dardenne 1999). Finalmente, se considera que el estudio integrado del SaLAM contribuye al mejor esclarecimiento de las correlaciones tectonoestratigráficas entre la CSL, mejor conocida, con los restantes registros contemporáneos de la CLM y los remanentes intermedios ya que pueden establecerse controles tectónicos e historias volcanosedimentarias comunes a todos ellos.

Agradecimientos Este trabajo se ha realizado a partir de investigaciones conjuntas que contribuyen a los proyectos "O Cretáceo Superior continental sedimentar da parte Austral da América do Sul" (FAPESP-Brasil) y TX 015 (UBACyT, Argentina). Se agradece a las Universidades de Buenos Aires y de la República y a las autoridades de la Administración Nacional de Combustibles, Alcoholes y Portland (ANCAP) el apoyo brindado. A los colegas Enrique Masquelín, que colaboró en el reconocimiento inicial de los filones fluoríticos en la Mina Florencia y en la obtención de su bibliografía, y Mario Torterolo por sus importantes comentarios con relación a las anomalías gravimétricas. A dos árbitros anónimos de RBG que mejoraron substancialmente la comprensión de este trabajo.

\section{Referências}

Bosse H.R., Gómez-Rifas C.G. Mari C 1982. Estudio geológico de la mina de fluorita «Florencia», Departamento de Maldonado, Uruguay. DINAMIGE - Instituto Federal de Geociencias y Materias Primas (Hannover), 25 p. Informe inédito.

Bossi J. 1978. Recursos minerales del Uruguay. Montevideo, Editorial Daniel Aljanati, $300 \mathrm{p}$

Bossi J. \& Navarro R. 1991. Geología del Uruguay. Montevideo, Universidad de la República - Dpto. Publicaciones, $970 \mathrm{p}$

Bossi J. \& Campal N. 1992. Magmatismo y tectónica transcurrente durante el Paleozoico inferior en el Uruguay. In: Gutiérrez Marco J.G, Saavedra J., Rábano I. (eds.) Paleozoico inferior de Ibero-América, España, Universidad de Extremadura, 343356.

Bossi J., Ferrando L.A., Montaña J., Campal N., Morales H., Gancio F., Schipilov A., Piñeyro D Sprechmann P., 1998 . Carta Geológica del Uruguay, escala l/500.000. Montevideo Edición Geoeditores SRL (CD-ROM)

Caorsi J.H. \& Goñi J.C. 1958. Geología Uruguaya. Montevideo, Instituto Geológico del Uruguay, 73p. (Boletín $\mathrm{N}^{\circ} 37$ )

Conceição J.J. de J., Zalán P.V., Wolff S. 1988. Mecanismo, evolução e cronologia do rift Sul-atlântico. Boletim Geociências Petrobras, 2 (2/4): 255-265.

Cobbold P.R., Szatmari P., Lima C., Rossello E.A. 1996. Cenozoic deformation across South America: Continent-wide data and analogue models. In: ORSTOMGéosciences Rennes, Saint Maló - Francia, International Simposium on Andean Geodynamics, 1: 21-24.

Christie-Blick N. \& Biddle K.T. 1985. Deformation and basin formation along strike-slip faults. In: Biddle K.T., Christie-Blick, N, (eds.). Strike-slip deformation, basin formation and sedimentation. Tulsa, Society of Economic Paleontologists and Mineralogists, Special Publication 37, 1-34

Dalla Salda L., Bossi J., Cingolani C. 1988. The Rio de La Plata cratonic region Southwestern Gondwanaland. Episodes, $1 /$ (4): 263-269.

Dardenne M.A. 1999. Os recursos minerais do Cretáceo no Brasil. In: UNESP/SBG, Serra Negra - Brasil, $5^{\circ}$ Simpósio sobre o Cretáceo do Brasil - $1^{\circ}$ Simposio sobre el Cretácico de América del Sur, Actas, 249-253.

Dardenne M.A., Ronchi L.H., Bastos-Neto A.C., Touray J.C. 1997. Geologia da fluorita. Os distritos de fluorita brasileiros. In: Schobbenhaus C., Queiroz E.T., Coelho C.E.S. (eds.) Principais depósitos minerais do Brasil. DNPM/CPRM, IVB, 479-507.

França A.B., Milani E.J., Schneider R.L., López-Paulsen O., López J.M., Suárez-Soruco R., Santa Ana H. de, Weins F., Ferreiro O., Rossello E. A., Bianucci E.H., Aramayo-
Flores R.F., Vistalli M.C., Fernández-Seveso F.A., Fuenzalida R.P., Muñoz N. 1996. Phanerozoic correlation in Southern South America. In: Tankard A.J., Suárez-Soruco R., Welsink H.J. (eds). Petroleum basins of South America. Tulsa, American Association of Petroleum Geologists, Memoir 62, 129-161.

Frostick L.E. \& Steel R.J. 1993. Tectonic signatures in sedimentary basin fills: An overview. Oxford, Blackwell, International Association of Sedimentologists, Special Publication, $\mathrm{N}^{\circ} 20,1-9$

Fontana R. L. 1990. Desenvolvimento termomecânico da Bacia de Pelotas e parte sul da Plataforma de Florianópolis. In: Raja Gabiglia G.P. \& Milani E.J. (eds.) Origem e evoluf̧áo de Bacias Sedimentures. Rio de Janeiro, PETROBRAS, 377-400.

Gómez-Rifas C.G. \& Pérez-Peirano M. 1992. El Cretácico del Uruguay y sus recursos minerales. In: IUGS-UNESCO/ Buenos Aires, Simposio de Recursos Minerales y Energéticos del Cretácico de América Latina, Actas, 185-210.

Gómez-Rifas C.G. \& Masquelin-Arcelus H.C. 1996. Petrología y geoquímica de las rocas volcánicas cretácicas del Uruguay. In: AGA/Buenos Aires - Argentina, XIII ${ }^{\circ}$ Congreso Geológico Argentino y III $^{\circ}$ Congreso de Exploración de Hidrocarburos, Actas, III: 635-652.
Harding T.P. 1990 . Identification of wrench faults using subsurface structural data: Criteria and piffalls. American Association Petroleum Geologists Bulletin 74 (10), 1590 and pitfa 1609 .

Introcaso A. \& Huerta E. 1982. Interpretación del exceso de gravedad en Uruguay. In: Buenos Aires, Argentina/ $5^{\circ}$ Congreso Latinoamericano de Geología, Actas, IV: 87 104.

Leeder M.R. \& Gawthorpe R.L. 1987. Sedimentary models for extensional tilt-block/halfgraben basins. In: Coward M.P., Dewey J.F., Hancock P.L. (eds.) Continental extensional tectonics. Geological Society Special Publication 28, 139-152.

Mann P., Hempton M.R, Bradley D.C., Burke K. 1983. Development of pull-apart basins. Journal of Geology, 91 : $529-554$

Muzio R. \& Artur C. 1998. Petrografía y geoquímica del Macizo alcalino Valle Chico, Depto. de Lavalleja, Uruguay: resultados preliminares. In: SUG/Punta del Este, II Congreso Uruguayo de Geología, Punta del Este - Uruguay, Actas, 145-150.

Muzio, R. Peel E., Artur C. 1999. New geochronological data of the Valle Chico alkaline massif (Uruguay), using U-Pb and Sm-Nd systematics. In: AGA/Córdoba - Argenmassif (Uruguay), using U-Pb and Sm-Nd systematics. In: AGA/Cordoba - Argen-
tina, II Simposio Sudamericano de Geología Isotópica, Extended abstracts (CD-

Naylor M.A., Mandl G., Supesteijn C.H.K. 1986. Fault geometries in basement-induced wrench faulting under different initial stress states. Joumal of Structural Geology, 8 (7): 737-752

Nielsen T.H. \& Sylvester A.G. 1995. Strike-slip basins. In: Busby C.J. \& Ingersoll R.V Tectonics of sedimentary basins. Cambridge, Blackwell Science, $549 \mathrm{p}$

Nurnberg D. \& Muller R. D. 1991 . The tectonic evolution of the South Atlantic from Late Jurassic to Present. Tectonophysics, 191:27-53.

Peate D.W. 1997. The Paraná-Etendeka Province. In: Mahoney J.J. \& Coffin M.F. (eds) Large igneous provinces, Continental: oceanic and planetary flood volcanism. American Geophysical Union, Geophysical Monograph 100, $217-245$.

Pirelli H. 1999 Contribución al conocimiento de la Formación Valle Chico, Uruguay. In: UNESP/SBG, Serra Negra - Brasil, $5^{\circ}$ Simpósio sobre o Cretáceo do Brasil y $1^{\circ}$ Simposio sobre el Cretácico de América del Sur, Anais, 433-437.

Preciozzi F., Spoturno J., Heinzen W., Rossi P. 1985. Carta geológica del Uruguay; l:500.000. Montevideo, DINAMIGE, $90 \mathrm{p}$.

Rabinowitz P.D. \& LaBreque J.L. 1979. The Mesozoic South Atlantic Ocean and evolution of its continental margins. Journal of Gerphysical Research, 84: 5973-6002.

Ramsay J.G. \& Huber M.I. 1983. The techniques of Modern Structural Geology. Volume 1: Strain analysis. London, Academic Press, $307 \mathrm{p}$.

Rossello E.A. \& Mozetic M.E. 1999. Caracterización estructural y significado geotectónico de los depocentros Cretácicos continentales del Centro-Oeste Ârgentino. In: UNESP/ SBG, Serra Negra - Brasil, $5^{\circ}$ Simpósio sobre o Cretáceo do Brasil y $1^{\circ}$ Simposio sobre el Cretácico de América del Sur, Anatis, 107-113.

Rossello EA. Santa Ana H. de, Veroslavsky G. 1999a. Rasgos transtensivos dextrales JuroCretácicos en la Cuenca Santa Lucía (Uruguay) y sus posibles reactivaciones Andinas. In: AGA, Salta - Argentina, XIV ${ }^{\circ}$ Congreso Geológico Argentino, Actas Andinas. In:

Rossello E.A., Santa Ana H. de, Veroslavsky G. 1999b. El Lineamiento Santa Lucía-AiguáMerín (Uruguay): Un rifting transtensivo Mesozoico abortado durante la apertura Atlántica? In: UNESP/SBG, Serra Negra - Brasil, $5^{\circ}$ Simpósio sobre o Cretáceo do Brasil y $1^{\circ}$ Simposio sobre el Cretácico de América del Sur, Anais: 443-448.

Rossello E.A., Santa Ana H. de, Veroslavsky G. 2000. La Cuenca Santa Lucía (Uruguay): tina (en prensa). 
Santa Ana H. de. 1989. Consideraciones tectónicas y deposicionales de la cuenca del Norte Uruguaya. ARPEL, Boletín Técnico, 18 (4): 319-339.

Santa Ana H. de \& Ucha N. 1994. Exploration perspectives and hydrocarbon potential of the uruguccon sedimentary bavins. Montevideo ANCAP 98p. (informe interno) the uruguayan sedimentary basins. Montevideo, ANCAP, 98p. (informe interno) Lucia (Uruguai): evolução tectônica e sedimentar. Geociências, 13 (1): 37:52.

Servicio Geográfico Militar 1973. Carta Gravimétrica Provisoria de la República Oriental del Uruguay, a escala $1: 1,000.000$, Montevideo, Rep. Oriental Uruguay.

Sprechmann P., Bossi J., Da Silva J. 1981. Cuencas del Jurásico y Cretácico del Uruguay. In: Buenos Aires - Argentina, Cuencas Sedimentarias del Jurásico y Cretácico de América del Sur. Comité Sudamericano del Jurásico y Cretácico, 1:239-270.

Thurston D.K. \& Theiss L.A. 1991. Identification of wrench faults using subsurface structural data: Criteria and pitfalls: Discussion. American Association of Petroleum Geologists Bulletin 75 (11): $1779-1781$.

Turner S.P., Regelous M., Kelley S., Hawkesworth C.J., Mantovani M.S.M. 1994. geochronology. Earth and Planetary' Science Letters, 121:333-348.

Uliana M.A. \& Biddle K.T. 1988. Mesozoic-Cenozoic paleogeographic and geodynamic evolution of Southern South America. Revista Brasileira de Geociências, 18 (2): evolution
Urien C.M., Zambrano J.J., Yrigoyen M.R. 1995. Petroleum basins of southern South America: an overview. In: Phanerozoic correlation in Southern South America. In: Tankard A.J., Suárez-Soruco R., Welsink, H.J (eds.) Petroleum basins of South America. Tulsa, American Association of Petroleum Geologists, Memoir No62, 6377.

Veroslavsky G. 1099 Geología da Bacia de Santa Lucia - Uruguai. Instituto de Geociências e Ciências Exatas, Universidade Estadual Paulista, São Paulo, Tese de Doutoramento, $152 \mathrm{p}$

Woodcock N H \& Schubert C. 1994. Continental strike-slip tectonics. In: Hancock P.L (ed.), Continental Deformation. Oxford, Pergamon Press, 251-263.
Manuscrito A-1142

Recebido em 25 de janeiro de 2000

Revisão dos autores em 10 de julho de 2000 Revissão aceita em 15 de julho de 2000 